Nicholai Studies, Vol. I, No. 1 (2021): 218-219.

DOI: https://doi.org/10.46825/nicholaistudies/ns.2021.1.1.218-219

\title{
Peer Reviewer Instructions
}

The Nicholai Studies primarily publishes original scientific papers on theological and religious subjects. By cultivating an interdisciplinary approach, the journal is open for works based on research in other social sciences and humanities, insofar as they correspond to the topic of the journal. The journal is also open for scientific papers and review articles based on research in other sciences and humanities - like philosophy, sociology, philology, literature, history, historiography, archival research, etc. - as long as they correspond with the topic of the journal. Nicholai Studies also publishes relevant archival and documentary material, with accompanying studies and notes as well as bibliographies, shorter notes, reviews, comments, and reviews of new publications.

Nicholai Studies primarily publishes articles in English and Serbian language. Every article published in Nicholai Studies is reviewed anonymously and two times before being published.

The Editorial Board of the Nicholai Studies holds to very high ethical and academic standards in the publication of scientific papers (see Ethical and Scientific Standards bellow, also available at https://nicholaistudies.org/en/ethical-and-scientific-standards). 


\section{The Process of Reviewing}

After receiving the paper, the Editorial Board of the Nicholai Studies will first decide whether the work fits the profile of the journal and whether it should be sent for review. If the Editorial Board evaluates the paper as corresponding to the profile of the journal, the paper is then being submitted to two reviewers. The names of reviewers are not and will not be known to the authors and all communication takes place through the Editorial Board. Reviewers receive the paper without the authors' names or other information that would reveal the identity of the author.

If the article is unconditionally accepted by both reviewers, the work is accepted for publication. If it is rejected by both reviewers, the work will not be published. If a reviewer or both reviewers asked for the article to be finished, the author will be introduced with requirements. After corrections/modifications, the article will be resubmitted to the reviewer/reviewers. If the reviewers estimate that the conditions are met, the paper will be published. If one reviewer conditionally or unconditionally evaluates the paper as positive and the other as negative, then a third reviewer will be engaged, and his review will be final.

Peer Review Form is available at the website of Nicholai Studies at https://nicholaistudies.org/en/peer-reviewer-instructions. 